\title{
Isolation and antioxidative properties of phenolics-saponins rich fraction from defatted rice bran
}

\begin{abstract}
The study presents a protocol for the preparation of phenolics-saponins rich fraction (PSRF), a new active nutraceutical from defatted rice bran followed by the determination of its antioxidant properties. PSRF was prepared by employing a simple alcoholic fractionation procedure on the crude alcoholic extract (CAE) of defatted rice bran. PSRF was found to be significantly higher in the contents of total phenolic, saponin, and steroidal saponin than CAE and its counterpart, aqueous fraction $(\mathrm{AqF})(\mathrm{p}<0.05)$. Except for iron chelating activity, PSRF exhibited notably higher activity than CAE and AqF in all antioxidant activity assays performed ( $\mathrm{p}<0.05)$. HPLC-DAD analysis revealed that PSRF contained substantially higher amounts of gallic acid, 4-hydroxybenzoic acid, caffeic acid, p-coumaric acid, and ferulic acid than $\mathrm{CAE}$ and $\mathrm{AqF}(\mathrm{p}<0.05)$. In conclusion, alcoholic fractionation of CAE simultaneously concentrated the phenolic compounds and saponins into PSRF, thus contributed to its higher antioxidant activity. Due to its elevated antioxidant properties, PSRF may be recommended for investigation as an active ingredient in the nutraceutical, functional food, and natural food preservative formulations. This is also the first report suggesting defatted rice bran as a potential and sustainable source of saponins.
\end{abstract}

Keyword: Saponins; Phenolic compounds; Defatted rice bran; Antioxidant activity 